\title{
Reconfiguração do horário de trabalho para turnos de 12h: que impactos na saúde?
}

\author{
Redefining work schedules for 12-hour shifts: which \\ health impacts?
}

\section{Reconfiguración de la jornada laboral para turnos de 12 horas: ¿qué impactos en la salud?}

Liliana Cunha

Daniel Silva ${ }^{2}$

Marta Santos $^{3}$

Cláudia Pereira ${ }^{4}$

Marianne Lacomblez ${ }^{5}$

\begin{abstract}
RESUMO: Num contexto normativo que amplia a margem para configuração de horários de trabalho ao abrigo de um "regime de adaptabilidade", acentua-se o debate sobre os turnos de $12 \mathrm{~h}$, em virtude de uma conjuntura que mobiliza mais trabalhadores para tais horários, bem como das evidências de impactos na saúde. A partir da análise da mudança de um horário $3 \times 8 \mathrm{~h}$ para 2x12h numa empresa portuguesa, este estudo explora os fatores e condições considerados de risco agravado em jornadas de $12 \mathrm{~h}$. Os resultados revelam que os trabalhadores, no confronto com os constrangimentos do trabalho nestes horários, constroem estratégias de preservação de si e do coletivo, ainda que estas estratégias comportem custos para a saúde. A ponderação (no imediato e a longo prazo) que cada trabalhador faz sobre a sustentabilidade do trabalho em $12 \mathrm{~h}$ convoca, simultaneamente, para debate: as especificidades do conteúdo de trabalho, a fase do percurso profissional e as suas exigências concretas, e os imperativos de conciliação com a vida fora do trabalho.
\end{abstract}

Palavras-chave:Horários.Trabalho por turnos.Saúde.Estratégias de regulação.

\footnotetext{
ABSTRACT:The normative context that enlarges the scope to shape work schedules under an "adaptability regime" has sharpened the debate about 12-hour shifts, given a conjuncture that leads more workers into such schedules and given the evidence of the impacts on health. Based on the

1 Faculdade de Psicologia e de Ciências da Educação da Universidade do Porto. 1cunha@fpce.up.pt

2 Centro de Psicologia da Universidade do Porto. danielsilva@fpce.up.pt

3 Faculdade de Psicologia e de Ciências da Educação da Universidade do Porto. marta@,fpce.up.pt

4 Centro de Psicologia da Universidade do Porto. cpereira@fpce.up.pt ;

5 Faculdade de Psicologia e de Ciências da Educação da Universidade do Porto. lacomb@fpce.up.pt
} 
analysis of the change from a $3 \times 8 \mathrm{~h}$ schedule into a $2 \times 12 \mathrm{~h}$ schedule in a Portuguese company, this study explores the factors and conditions considered to be aggravated risks in 12-hour journeys. The findings indicate that the workers, when confronted with the constraints of the work in these schedules, build preservation strategies for themselves and for the group, though such strategies entail health costs. The deliberation each worker does (immediately and in the long term) about the sustainability of the work in 12 hours calls simultaneously for debate: the specificities of the work content, the stage of the professional path and its specific demands, and the imperatives of reconciliation with life outside work.

Keywords: Schedules.Shift work. Health.Regulationstrategies.

RESUMEN: En un contexto normativo que amplía el margen para el ajuste de la jornada laboral en virtud de un "régimen de adaptabilidad", se intensifica el debate sobre los turnos de 12 horas, debido a una coyuntura que moviliza más trabajadores para esos horarios, así como a las evidencias de los impactos en la salud. A partir del análisis del cambio de un horario de 3 x 8 horas para 2 x 12 horas en una empresa portuguesa, este estudio explora los factores y condiciones que se consideran de riesgo agravado en jornadas de 12 horas. Los resultados revelan que los trabajadores, al enfrentarse con las limitaciones del trabajo en estos horarios, construyen estrategias de autopreservación y de preservación del colectivo, aunque estas estrategias conlleven costes para la salud. La ponderación (en lo inmediato y en el largo plazo) que cada trabajador hace sobre la sostenibilidad de trabajar 12 horas llama al mismo tiempo para el debate: los detalles del contenido del trabajo, la fase de la carrera y sus requisitos específicos, y los requisitos de reconciliación con la vida fuera del trabajo.

Palabras clave: Horarios. Trabajo por turnos. Salud. Estrategias de regulación.

\section{INTRODUÇÃO}

\subsection{O recurso normalizado a horários atípicos}

Na Europa, e sobretudo após a definição da Diretiva “Tempo de Trabalho", em 1993, e das suas subsequentes revisões (em 2000 e 2003), verifica-se uma diminuição do número de horas de trabalho, mas, em paralelo, assiste-se cada vez mais à diversificação dos mecanismos que ditam a sua intensificação ${ }^{1,2,3}$.Um certo recuo do que então era norma tem, de facto, ganho protagonismo com os horários ditos "atípicos"4. A implementação destes horários adquire maior expressão, nos últimos anos, como o indicam os dados mais recentes dos inquéritos europeus sobre condições de trabalho ${ }^{5,6}$. Nesta tendência, assinala-se, igualmente, o ressurgimento dos horários que prolongam o período de trabalho além das 8 horas usuais (até $12 \mathrm{~h}$ por jornada) ${ }^{4,7}$. Oalargamento da duração do turno de trabalho é favorecido por regulações europeias e nacionais cada vez mais flexíveis.A mobilização flexível dos trabalhadores torna-se modelo dominante ${ }^{8}$, no seio de uma certa desconstrução, quer da duração do trabalho, quer da sua organização nas $24 \mathrm{~h}$ do dia, por contraponto com o registo tradicional de $8 \mathrm{~h}$. 
A discussão destas questões suscita tensões, seja do ponto de vista científico - pelo património de saberes já constituídos sobre os efeitos negativos na saúde e bem-estar destes horários ${ }^{9,10,11-}$ ou do ponto de vista dos próprios trabalhadores (pela questão do emprego, da remuneração, da repartição do trabalho). Mas, assim o é também, do ponto de vista legal, quando as políticas de flexibilidade conduzem a uma subordinação crescente das exigências humanas à rentabilização dos equipamentos $^{12}$, fazendo prevalecer determinados critérios de legitimidade desta opção de horários relativamente a outros, como o da segurança e saúde dos trabalhadores.

\subsection{Os turnos $2 \times 12 \mathrm{~h}$ no quadro de um "regime de adaptabilidade"}

Em Portugal, a organização temporal em $12 \mathrm{~h}$ tem sido mantida por um ordenamento jurídico que contempla um regime dito de "adaptabilidade" (Artigos 203 - 207 da lei laboral portuguesa), em que o Período Normal de Trabalho (PNT) pode ser definido em termos médios, tendo em conta um período de referência não superior a 12 meses. Assim, o PNT pode ser definido, em termos médios, até mais $4 \mathrm{~h}$ por dia, além do horário regular, e com uma duração máxima de $60 \mathrm{~h}$ por semana.

Mas, "adaptabilidade" de quê e de quem? Por um lado, adaptabilidade do horário em função dos fluxos do mercado e das necessidades de produção. A transição dos sistemas 3x $8 \mathrm{~h}$ para 2x12h é tida pelas empresas como um epíteto para o aumento da produtividade, em virtude da diminuição do número de passagens de turno ou do hipotético maior controlo dos riscos associados a modos operatórios diferenciados, próprios às sucessivas equipas de trabalho ${ }^{13,14}$.

Do ponto de vista dos trabalhadores, os turnos de $12 \mathrm{~h}$ poderão ser perspetivados como resposta à necessidade de um melhor compromisso para atender às exigências da vida fora do trabalho. Tal é traduzido sob a forma de uma perceção de "ganho para si" fora da empresa, ou de uma "libertação" dos condicionalismos resultantes do sistema $3 \times 8 \mathrm{~h}^{15}$.

Não obstante estes argumentos, que realçam sobretudo as vantagensperspetivadas pelos protagonistas do regimeem $2 \times 12 \mathrm{~h}$, diferentes contributos no domínio da Ergonomia da Atividade e da Psicologia do Trabalho ${ }^{16,17}$ têm evidenciado as exigências que tal horário entrevê, no confronto dos trabalhadores com as discordâncias que acabam por surgir entre as exigências temporais do trabalho, as características do funcionamento humano e os ritmos sociofamiliares ${ }^{18}$.

A adaptabilidade não depende somente de uma preconização legal, até porque não se mantém externa à atividade e aos trabalhadores. A conceção do ser humano trabalhador que lhe subjaz tem implícita a estabilidade do funcionamento humano, tanto de dia como de noite. E ainda que as jornadas de $12 \mathrm{~h}$ sejam legitimadas pela compensação de mais folgas, não existe um corpo sem memória dos efeitos da intensificação do trabalho.

\subsection{Riscos do trabalho em $12 \mathrm{~h}$ e efeitos na saúde}

Os trabalhadores abrangidos por este tipo de horários frequentemente experienciam situações 
de destabilização/desequilíbrio, determinadas pelas discordâncias entre as exigências temporais do trabalho, as características do funcionamento humano, e os seus ritmos sociofamiliares. Estas situações produzem um redimensionamento das exigências do trabalho, o que pode contribuir para a deterioração do estado de saúde e bem-estar, bem como para a destabilização da organização da vida fora do trabalho ${ }^{19,20}$.

A alternância "mais frequente" entre períodos noturnos e diurnos de trabalho em $12 \mathrm{~h}$ representa uma condição fisiológica desfavorável para o corpo, colocando os trabalhadores em cenários em que tentam "habituar-se" o mais possível ao desalinhamento dos ritmos circadianos a que estão submetidos ${ }^{21}$. Na saúde, entre as consequências mais reportadas, destacam-se problemas de sono, agravamento das queixas músculo-esqueléticas, problemas digestivos e gastrointestinais, fadiga crónica e alteração de funções cognitivas relativas à atenção, vigilância e memória a curto $\operatorname{prazo}^{22,23,24,25}$.

\subsection{Objetivos do estudo}

Partindo do pedido de uma empresa portuguesa de produção de bebidas - de análise dos impactos percebidos pelos trabalhadores da área de enchimento após a reconfiguração do horário de trabalho, de turnos de $8 \mathrm{~h}$ para $12 \mathrm{~h}$-o objetivo deste estudo consistiu em analisar a atividade de trabalho concreta e a perceção dos trabalhadores face aos riscos do trabalho no novo horário face ao horárioanterior. $\mathrm{O}$ estudo confere ainda visibilidade às estratégias que desenvolvem com o intuito de assegurar, simultaneamente, o funcionamento contínuo das instalações produtivas, e a gestão das variações circadianas, a acumulação da fadiga, ou os conflitos de temporalidades que marcam a conciliação das diferentes dimensões da vida.

\section{MÉTODOS}

\subsection{Contexto da pesquisa}

A mudança de horário na empresa resultou da iniciativa dos trabalhadores, sob o argumento de que o horário de $12 \mathrm{~h}$ lhes concederia outra margem de manobra para a conciliação do trabalho com o ritmo da vida fora do trabalho.A decisão da empresa de implementar esta nova forma de organização temporal do trabalho, após uma fase significativa de negociações, foi determinada como "temporária", e caracterizada como "experimental".

Com o intuito de consubstanciar esta decisão, foi dirigido um pedido de análise à nossa equipa de investigação ${ }^{6}$ sobre este processo de transição de horários, e sobre o ponto de vista dos trabalhadores relativamente à experiência em $12 \mathrm{~h}$.

Nesta empresa, a laboração contínua é agora assegurada por 4 equipas, num total de 92 trabalhadores, alternando entre turnos de $12 \mathrm{~h}$ (08h-20h; 20h-08h). Por mês, são realizadas por

6 Psicólogos do trabalho da Faculdade de Psicologia e de Ciências da Educação da Universidade do Porto. 
$95 / /$

cada trabalhador 14 jornadas de $12 \mathrm{~h}$ ( 7 dias +7 noites), sendo intercaladas por blocos de dias de folga (14 dias por mês). Tanto de dia como de noite, os trabalhadores cumprem até 3 jornadas consecutivas, antes do período de descanso.

O estudo contemplou a participação de todos os trabalhadores da área de produção, ainda que nem todos tenham participado em todas as etapas de investigação, como a seguir melhor se explicita.

\subsection{Etapas de investigação}

Para a análise da alteração do horário de trabalho nesta empresa foi privilegiada uma abordagem situada, possível através da análise ergonómica do trabalho ${ }^{16}$, procurando conhecer o conteúdo do trabalho, os constrangimentos em jogo, os riscos agravados com a nova configuração horária, ou as estratégias construídas no seio das equipas, tendo em vista o compromisso entre as exigências da atividade e a gestão da saúde ${ }^{26}$. Neste sentido, no plano de investigação, foram cruzadas duas fontes principais de dados: análise qualitativa (análise da atividade com recurso a observações e entrevistas) e quantitativa (questionário).

Num primeiro momento, a análise do trabalho foi sustentada por um conjunto de observações em contexto real e no registo de verbalizações dos trabalhadores de todas as linhas de produção. A partir deste corpo de conhecimentos sobre a atividade, a fase seguinte envolveu a realização de entrevistas, junto de uma amostra de trabalhadores $(N=20)$ de todas as linhas de produção e equipas de trabalho.

O terceiro momento de investigação implicou a construção de um questionário, a ser preenchido online. O questionário foi enviado a todos trabalhadores das linhas de produção $(N=92)$, tendo sido registada uma taxa de resposta de 66\% (média etária de 37 anos; antiguidade média de 12.5 anos).A avaliação do novo horário sustentada neste questionário foi efetuada quando decorriam nove meses após a implementação da escala $2 \times 12 \mathrm{~h}$, num momento em que havia já uma experiência de trabalho constituída neste horário.

\section{RESULTADOS}

\subsection{A organização da escala $2 \times 12 h$ vs. $3 \times 8 h$ : o que mudou?}

O anterior horário $3 \times 8 \mathrm{~h}$ estava organizado num ciclo de 84 dias, onde era registada a rotação entre turnos da manhã (07:30h-15:30h), turnos da tarde (15:30h-23:30h), turnos da noite (23:30h-07:30h), e períodos de folga. Assim, por cada ciclo, a escala assumia a seguinte configuração ${ }^{7}$ : $5 \mathrm{~N}-\mathrm{F}-5 \mathrm{~T}-\mathrm{F}-$ $5 \mathrm{M}-\mathrm{F}-3 \mathrm{~N}-3 \mathrm{~F}-3 \mathrm{~T}-\mathrm{F}$.

Atualmente, como foi já referido, a escala em vigor 2x12h está organizada num ciclo de 28 dias, com a seguinte alternância entre turnos diurnos (08h-20h), turnos noturnos (20h-08h) e períodos de

7 N - Noite; T - Tarde; M - Manhã; F - Descanso/folga. 
folga: 3F-2D-2F; 2N-3F-2D; D-F-2N-3F; F-2D-F-3N.

Na Tabela 1, é apresentada uma comparação entre estas duas escalas de trabalho, para os trabalhadores.

Tabela 1.Análise comparativa das duas escalas de trabalho (3x $8 \mathrm{~h} v s .2 \times 12 \mathrm{~h})$

\begin{tabular}{lcc}
\hline Número médio de horas de trabalho por semana & $\mathbf{3 x 8 h}$ & $\mathbf{2 x 1 2 h}$ \\
Número máximo de turnos consecutivos (diurnos ou noturnos) & 52 & 42 \\
\hline Número de turnos de trabalho por mês & 5 & 3 \\
Número de folgas por mês & 23 & 15 \\
Número máximo de folgas consecutivas & 3 & 15 \\
Número de fins-de-semana livres por ano & 13 & 4 \\
\hline Número de turnos de trabalho por ano & 274 & 182 \\
\hline
\end{tabular}

Com a implementação da escala $2 \times 12 \mathrm{~h}$ registou-se uma diminuição do número de turnos de trabalho por ano, ainda que, em termos médios, sejam trabalhadas o mesmo número de horas por semana (42h).

\subsection{Os riscos do trabalho em $12 \mathrm{~h}$ e efeitos na saúde}

Após 9 meses de trabalho em $2 \times 12 \mathrm{~h}$, a maioria dos trabalhadores $(80.3 \%)$ considera que a aplicação do horário $2 \times 12 \mathrm{~h}$ deveria ser estendida além do período experimental. Tal posicionamento favorável era justificado pela maior disponibilidade que o novo horário permite (nomeadamente pela concentração dos períodos de descanso/folgas) para atender às exigências da vida fora do trabalho; e ainda pela diminuição do número de turnos de trabalho consecutivos (no máximo três, em $2 \times 12 h)$. O que fica, ainda assim, na penumbra desta decisão?

Os trabalhadores entreveem uma exposição diferenciada aos riscos, tanto físicos como resultantes da organização do trabalho em $2 \times 12 \mathrm{~h}$, comparativamente com o horário anterior ( $3 \times 8 \mathrm{~h})$. Na Tabela 2, são identificadas as condições e exigências consideradas de risco, e que agora são percebidas como "amplificadas" em $2 \times 12 \mathrm{~h}$, por uma percentagem significativa de trabalhadores.

Tabela 2.Percentagem de trabalhadores que sinaliza o agravamento dos riscos em $2 \times 12 \mathrm{~h}$ 


\begin{tabular}{|c|c|}
\hline Fatoresfísicos & $N(\%)$ \\
\hline Ruído & $45(73.8 \%)$ \\
\hline Permanecer muito tempo de pé & $41(67.2 \%)$ \\
\hline Ter de subir e descer com frequência & $33(54.1 \%)$ \\
\hline Ter de fazer esforços físicos intensos & $32(52.5 \%)$ \\
\hline Fatores associados à organização do trabalho & $N(\%)$ \\
\hline Ter um número de elementos insuficiente na equipa & $45(73.8 \%)$ \\
\hline Acompanharostrabalhadorestemporários & $37(60.7 \%)$ \\
\hline Ter de trabalhar a um ritmo intenso & $31(50.8 \%)$ \\
\hline
\end{tabular}

Um dos casos mais ilustrativos deste redimensionamento do risco percebido é relativo ao ruído; isto é, o nível de ruído, não é superior ou inferior em $12 \mathrm{~h}$ em relação às $8 \mathrm{~h}$, mas o alargamento da exposição diária (mais $4 \mathrm{~h}$ por turno) redimensiona o constrangimento sentido pelos trabalhadores.

Por outro lado, sobre os impactos percebidos do horário de $12 \mathrm{~h}$ em interação com os riscos "amplificados", os trabalhadores perspetivam atualmente um agravamento dos problemas de saúde (cf. Tabela 3).

Tabela 3. Principais queixas de saúde reportadas, segundo o horário de trabalho $-N(\%)$

\begin{tabular}{lccc}
\hline & $\begin{array}{c}\text { Mais grave em } \\
3 x 8 h\end{array}$ & $\begin{array}{c}\text { Agravadoem } \\
2 x 12 h\end{array}$ & $\begin{array}{c}\text { Parece não ter relação } \\
\text { com o horário }\end{array}$ \\
$\begin{array}{l}\text { Problemas de sono } \\
\begin{array}{l}\text { Apatia e desencorajamento } \\
\text { generalizado }\end{array}\end{array}$ & $11(18 \%)$ & $15(24.6 \%)$ & $12(19.7 \%)$ \\
$\begin{array}{l}\text { Fadigageneralizada } \\
\text { Ansiedadeouirritabilidade }\end{array}$ & $12(19.7 \%)$ & $24(39.3 \%)$ & $7(11.5 \%)$ \\
$\begin{array}{l}\text { Doresmusculares } \\
\text { Doresnascostas }\end{array}$ & $2(3.3 \%)$ & $10(16.4 \%)$ & $9(14.8 \%)$ \\
\hline
\end{tabular}

Os resultados evidenciam uma perceção de agravamento das principais queixas associadas ao trabalho em $2 \times 12 h$, em especial as dores musculares. Este indicador está em linha com os principais fatores de risco considerados pelos trabalhadores (cf. Tabela 2): permanecer muito tempo de pé, ter de subir e descer com frequência ou fazer esforços físicos intensos. Por outro lado, a questão do desencorajamento/apatia generalizada é sinalizada como mais presente em $3 \times 8 \mathrm{~h}$ do que atualmente em $2 \times 12 \mathrm{~h}$, sendo que tal poderá ser justificado pela: diminuição do número de turnos consecutivos realizados numa semana de trabalho (cinco turnos em $3 \times 8 \mathrm{~h} v$ s. três turnos em $2 \times 12 \mathrm{~h}$ ); e, consequentemente, pela maior margem temporal agora possível para a gestão das exigências da 
vida fora do trabalho.

\subsection{O dia-a-dia em 12h: a (re)construção de estratégias de regulação}

A alteração do horário para $2 \times 12 \mathrm{~h}$ produz impactos na saúde, mas também na atividade de trabalho e na vida privada. A mediação destes impactos é suportada pela implementação de estratégias de regulação no trabalho, individuais e coletivas, sendo também relatadas um conjunto de regulações individuais fora do trabalho. Na Tabela 4 sistematiza-se algumas destas estratégias construídas em $12 \mathrm{~h}$, no trabalho - em especial no período noturno.

Com a alteração do horário de trabalho ocorreram de facto uma série de mudanças na regulação da atividade. Trabalhar $12 \mathrm{~h}$ por turno requer uma reelaboração da habilidade constante de adaptação às exigências, com a mobilização de estratégias de um compromisso possível redefinidas.

Tabela 4.Principais estratégias desenvolvidas face ao horário $2 \times 12 \mathrm{~h}$

\section{Regulação coletiva no trabalho}

- Novos ciclos de rotação nos próprios postos de trabalho, cuja sequência e ritmo são função das exigências particulares das máquinas (de 2 em 2 h numa máquina; de 6 em 6 h numa outra;...).

- Aumento da periodicidade e do número de pausas (variável de equipa para equipa, e se é turno diurno ou noturno: entre 2 e 4 pausas).

- Revezamento: de forma mais notória à noite (entre a $01 \mathrm{~h}$ e as $04 \mathrm{~h}$ ), em função dos níveis de sonolência e fadiga, a equipa reorganiza-se internamente de modo a permitir que um colega se ausente da linha por um curto intervalo.

- Preparar as tarefas: com o aproximar do fim do turno, certas tarefas não são inteiramente iniciadas, mas sim "preparadas", para os colegas que iniciarão o turno num estado de maior alerta e vigilância.

\section{Regulação individual: no trabalho}

- Antecipação das tarefas: em função do plano de produção, o trabalhador pode alocar as tarefas que exigem mais atenção para o início do turno.

- Regulação do ritmo nos períodos de maior cansaço e menor vigilância: o trabalhador pode aumentar ou diminuir o ritmo de produção ( $\mathrm{n}^{\mathrm{o}}$ de unidades) em função da maior ou menor proximidade com o objetivo produtivo e do seu estado (nível de vigilância e de fadiga).

\section{Regulação individual: vida fora do trabalho}

- Preparar as $12 \mathrm{~h}$ : antes de iniciar um turno noturno, assiste-se à "gestão" das fases do sono (i.e., não dormir de manhã e dormir o maior número de horas possíveis à tarde). Por outro lado, outras estratégias são mobilizadas na expectativa que o sono diurno seja menos interrompido (e.g., não ingerir líquidos com o aproximar do fim do turno).

- Após um turno noturno, algumas tarefas são realizadas antes dos trabalhadores irem dormir (e.g., levar os filhos à escola).

De um "tempo constrangido" pelas características do processo de trabalho e da sua organização temporal, observamos a ação concreta do coletivo, tendo em vista um "tempo (re)construído"19, no sentido de minimizar os custos associados aos turnos de $12 \mathrm{~h}$. Ainda que mais notoriamente expressas 
durante o período noturno ${ }^{20}$, e de forma diferenciada dependendo da idade e da experiência dos trabalhadores, tais estratégias possibilitam uma "redefinição de prioridades" a atingir, considerando a fase do plano de produção do turno, a composição da equipa, ou o estado de vigilância e o cansaço sentido (por si e pelos outros).

Finalmente, a análise conduzida nesta empresa tornou evidentes os modos como o "tempo dentro do trabalho"27, definido a partir do pressuposto da constância do funcionamento humano ao longo de 12h, "invade" o domínio da vida fora do trabalho. Deste modo, os dias de folga são investidos para a compensação da "dívida de sono", para recuperação do cansaço, mas também para preparar o corpo para as próximas $12 \mathrm{~h}$.

\section{4 "Trabalhar em 12h?" Sim, mas até quando?}

Quando a ponderação que é realizada sobre a viabilidade do horário $2 \times 12 \mathrm{~h}$ é tida num horizonte temporal mais amplo, como até à idade da reforma, as respostas dos trabalhadores revelaram-se menos unânimes (cf. Figura 1). Sobre se se imaginam a trabalhar no horário 2x12h até à idade da reforma, $50.8 \%(N=31)$ dos trabalhadores considera que não, e $49.2 \%(N=30)$ indica que sim.

Figura 1. Posicionamento dos trabalhadores face ao horário $2 \times 12 \mathrm{~h}$, na atualidade e perante a sua viabilidade até à reforma

\section{$\%$ de trabalhadores que deseja manter o horário $2 \mathrm{x} 12 \mathrm{~h}$ neste momento}

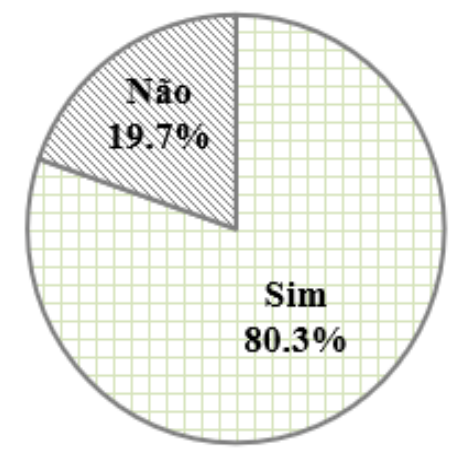

$\%$ de trabalhadores que se imagina no horário $2 \times 12 \mathrm{~h}$ até à idade da reforma

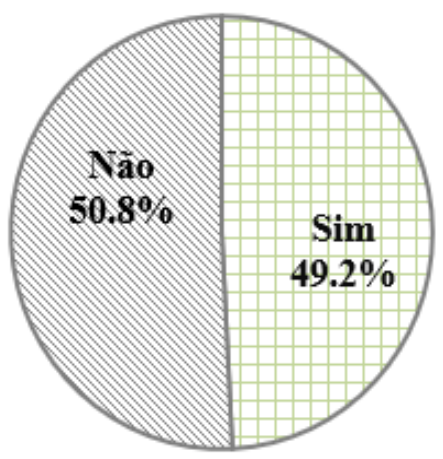

A organização temporal do trabalho em $12 \mathrm{~h}$ embora possa apresentar vantagens, para as empresas, e para os trabalhadores, redimensiona os riscos, como os resultados deste estudo sinalizam, reiterando-se assim a importância de não tratar os riscos ligados aos horários isoladamente, mas sim considerar os efeitos conjuntos das exigências do trabalho e das condições em que é exercido.

A arbitragem entre a preservação da saúde e a garantia de uma maior margem de manobra para a conciliação do trabalho com a vida social e familiar, porque a sua conciliação não se revela unânime, é incomensurável. A opção por um horário em $12 \mathrm{~h}$ pode ser particularmente útil em 
determinados momentos da vida dos trabalhadores, e quando os efeitos do trabalho na saúde mantêm uma expressão discreta, infrapatológica. Mas, a análise num horizonte temporal mais alargado revela que a realização do trabalho até à idade da reforma é menos consensual.

\section{CONCLUSÕES}

Um horário $2 \times 12 \mathrm{~h}$, quando analisado no quadro de uma atividade concreta, recentra o nosso olhar: percebemos que a "adaptabilidade" preconizada no discurso legal requer, na verdade, a construção de um compromisso de sustentabilidade, de si e de outros, na tentativa de assegurar, tanto quanto possível, a preservação da saúde. A ponderação entre as duas opções de organização temporal, no caso dos trabalhadores desta empresa, é arbitrada pelo que se percebe como "um mal menor"15: os ganhos na vida fora do trabalho não parecem compensar os custos maiores a que o trabalho nestes horários obriga.

O património de saberes de que dispomos atualmente no âmbito da Ergonomia e da Psicologia do Trabalho há muito alertam para o agravamento dos problemas de saúde, associados à intensificação do trabalho potenciada por horárioscada vez mais irregulares ${ }^{3}$. Eis o paradoxo que este estudo reforça também: apesar de a escala de $12 \mathrm{~h}$ aqui apresentada se encontrar enquadrada legalmente pelo que preconiza a "Diretiva Tempo de Trabalho", não é verdade que a experiência de trabalho neste quadro de organização temporal seja menos suscetível de riscos. Pelo contrário, os riscos são redimensionados no horário em $12 \mathrm{~h}$. Neste sentido, e com o intuito de minorar tanto quanto possível tal "redimensionamento", no âmbito da investigação realizada, os trabalhadores definiram um conjunto de sugestões de melhoria às condições de trabalho. Tais sugestões foram assumidas pela empresa enquanto recomendações a implementar a curto/médio prazo:

- $\quad$ pecaver as situações em que se verifica a ausência de um ou dois trabalhadores na equipa, adaptando o plano de enchimento ao número de trabalhadores presentes na linha;

- $\quad$ prever oficialmente um maior número de pausas durante os turnos de $12 \mathrm{~h}$;

- $\quad$ prevenir as situações de trabalho em isolamento (evitar trabalhar sozinho, tendo colegas a quem recorrer, em especial no período noturno).

A monitorização dos impactos do horário $2 \times 12 \mathrm{~h}$, e da sua sustentabilidade, beneficiaria de um plano de investigação longitudinal. De forma a colmatar esta limitação do estudo, a nossa proposta de ação junto da empresa consistiu na definição de um dispositivo de acompanhamento e avaliação, de cariz longitudinal, da evolução dinâmica dos efeitos dos tempos de trabalho, nos seguintes níveis de análise: saúde, considerando desde situações de patologia a queixas de alteração da arquitetura do sono; segurança, prevendo a monitorização dos riscos que determinam a intensificação do trabalho; e produção e qualidade, equacionando o ajustamento das metas definidas, face às temporalidades próprias do funcionamento humano. 
Contribuição dos autores:Todos os autores do artigo participaram nas diferentes etapas do estudo, designadamente, na conceção do estudo, na recolha de dados, nas análises conduzidas e na redação do manuscrito.

\section{REFERÊNCIAS BIBLIOGRÁFICAS}

1. Gollac M, Volkoff S, Wolff L. Les conditions de travail. Paris: La Découverte, 2014.

2. Piasna A. Scheduled to work hard: the relationship between non-standard working hours and work intensity among European workers (2005-2015). Human Resources Management Journal 2018; 28:167-181.

3. Volkoff S, Delgoulet C. L'intensification du travail, et l'intensification des changements dans le travail: quels enjeux pour les travailleurs expérimentés? Psychologie du Travail et des Organisations 2019; 25:28-39.

4. CostaG, Prunier-Poulmaire S, Barthe B. Horaires de travail et Société: quels constats, quelles évolutions en Europe et en France? In Actes du 48ième Congrès de la SELF, Ergonomie \& Société, Quelles attentes? Quellesréponses?, Paris, France, 2013.

5. Eurofound. Sixth European working conditions survey. Luxembourg: Publications Office of the European Union, 2017.

6. Eurofound. Working conditions on global perspective. Luxembourg: Publications Office of the European Union and International Labour Organization, 2019.

7. Barthe B. Les $2 \times 12 \mathrm{~h}$ : une solution au conflit de temporalités du travail posté? Temporalités 2009; 10:1-19.

8. Martinez E. Les salariés à l'épreuve de la flexibilité. Bruxelles: Éditions de l'Université de Bruxelles, 2010.

9. Bourdouxhe M, Quéinnec Y, Granger D, Baril H, Guertin S, Massicotte P, Levy M, Lemay F. Aging and shiftwork: the effects of 20 years of rotating 12-hour shifts among petroleum refinery operators. Experimental Aging Research 1999; 25:323-329.

10. Barthe B. La déstabilisation des horaires de travail. In A. Thébaud-Mony, P. Davezies, L. Vogel, \& S. Volkoff (Dirs.), Les risques du travail (pp. 223-232). Paris: La Découverte, 2015.

11. Marquié J-C. Le travail de nuit: conséquences sur le sommeil et les performances. Les Cahiers de l'Actif 2016; $482: 27-42$. 
12. Vogel L. Les surprises de la directive communautaire concernant certains aspects de l'aménagement du temps de travail. L'annéesociale 1997; 423-446.

13. Smith L, Folkard S, Tucker P, Macdonald I. Work shift duration: a review comparing 8 hour and 12 hour shift systems. Occup Environ Med 1998; 55: 217-229.

14. Knauth P. Extended work periods. Industrial Health 2007; 45: 125-136.

15. Cunha L, Silva D, Ferreira I, Pereira C, Santos M. When the working hours become a risk factor: the debate about $3 \times 8 \mathrm{~h}$ and $2 \times 12 \mathrm{~h}$ shifts. In P. Arezes, J. Baptista, M. Barroso, P. Carneiro, P. Cordeiro, N. Costa, R. Melo, A. Miguel, \& G. Perestelo (Eds.), Occupational Safety and Hygiene VI (pp. 239-244). London: CRC Press-Taylor \& Francis, 2008.

16. Lacomblez M, Teiger C. Ergonomia, formações e transformações. In P. Falzon (Ed.), Ergonomia (pp. 587-601). São Paulo: Blucher, 2007.

17. Guérin F, Laville A, Daniellou F, Duraffourg J, Kerguelen A. Understanding and transforming work: the practice of ergonomics. Lyon: ANACT, 2007.

18. Quéinnec Y, Teiger C, de Terssac G. Repères pour négocier le travail posté. Toulouse: Octarès, 2008.

19. Toupin C, Barthe B, Prunier-Poulmaire S. From constrained to constructed working time. In P. Falzon (Ed), Constructive Ergonomics (pp. 65-78). New York: CRC Press, 2015.

20. Cheyrouze M, Barthe B. Travail de nuit en 12 heures: un scénario de travail élaboré par les infirmiers dans un service de réanimation. Activités2018; 15:1-44.

21. Costa G.Shift work and health: current problems and preventive actions. Safety Health at Work 2010; 1(2): 112-123.

22. Prunier-Poulmaire S, Gadbois C, Volkoff S. Combined effects of shift systems and work requirements on customs officers. Scandinavian Journal of Work, Environment \& Health 1998; 24(3): 134-140.

23. Caruso C. Possible broad impacts of long work hours. Industrial Health 2006; 44: 531-536.

24. Tucker P, Folkard S. Working time, health and safety: a research synthesis paper. Geneva: International Labour Office, 2012.

25. Kecklund G,Axelsson J. Health consequences of shift work and insufficient sleep. The BMJ 
2016; 355: 1-13.

26. Barthe B, Gadbois C, Prunier-Poulmaire S, Quéinnec Y.Trabalhar em horários atípicos. In P. Falzon (Ed.), Ergonomia (pp. 97-109). São Paulo: Blucher, 2007.

27. Quéinnec Y. Horário. Laboreal 2007; 3(2): 90-91.

Artigo apresentado em: abril de 2019 Artigo aprovado em: junho 2019 Conflito de interesses: os autores declaram não haver conflito de interesses. Financiamento: não Suporte financeiro: os autores declaram também que o desenvolvimento deste manuscrito não teve qualquer apoio financeiro ou material. 